\title{
Tecnologia de enfermagem na prevenção da úlcera por pressão em pessoas com lesão medular
}

Nursing technology in the prevention of pressure ulcer in people with spinal cord injury

Tecnología de enfermería en la prevención de úlcera por presión en personas con lesión medular

\section{Rita Mônica Borges Studart', Elizabeth Mesquita Melo', Marcos Venícios de Oliveira Lopes", Islene Victor Barbosa"II, Zuila Maria de Figueiredo Carvalho"}

' Universidade de Fortaleza (UNIFOR). Departamento de Enfermagem. Fortaleza-CE, Brasil.

" Universidade Federal do Ceará (UFC). Faculdade de Farmácia, Odontologia e Enfermagem. Fortaleza-CE, Brasil.

III Universidade Federal do Ceará (UFC). Faculdade de Farmácia, Odontologia e Enfermagem.

Doutoranda do Programa de Pós-Graduação em Enfermagem. Fortaleza-CE, Brasil.

Submissão: 14/01/2010 Aprovação: 10/02/2011

RESUMO

Estudo transversal do tipo exploratório descritivo realizado em um hospital público referência em trauma no período de maio a outubro de 2008. Objetivou-se avaliar a tecnologia de enfermagem utilizando a Escala de Waterlow para prevenir a úlcera por pressão em pessoas com lesão medular. Foram avaliadas 60 pessoas com lesão medular utilizando-se a Escala de Waterlow, das quais 75\% apresentaram escore final de 28 pontos, com altíssimo risco para desenvolver úlcera por pressão. Após dez dias de internação, 48,3\% dos pacientes ainda não haviam recebido orientações preventivas, dos quais 68,3\% já apresentavam úlcera por pressão. A utilização da Escala de Waterlow permite a monitorização da assistência, sendo capaz de influenciar nos resultados clínicos.

Descritores: Enfermagem; Úlcera por pressão; Tecnologia; Escalas.

\section{ABSTRACT}

Cross-sectional and exploratory-descriptive study, conducted in a public hospital, of reference in trauma, in the period from May to October 2008. The objective was to evaluate the nursing technology using the Waterlow Scale to prevent pressure ulcers in people with spinal cord injury. Sixty patients with spinal cord injury were evaluated using the Waterlow Scale, of which $75 \%$ had final score of 28 points, with high risk for developing pressure ulcers. After ten days of hospitalization, 48,3\% of those patients had not received any preventive guidelines; $68,3 \%$ of them already had pressure ulcers. The use of the Waterlow Scale allows monitoring of assistance, being able to influence clinical outcomes.

Key words: Nursing; Pressure ulcer; Technology; Scales.

\section{RESUMEN}

Estudio transversal y exploratorio-descriptivo, realizado en un hospital público, de referencia en trauma, en el período de mayo a octubre de 2008. El objetivo fue evaluar la tecnología de enfermería utilizando la escala de Waterlow para prevenir las úlceras por presión en personas con lesión de la médula espinal. Sesenta pacientes con lesión de médula espinal fueron evaluados tilizando la escala de Waterlow, de los cuales 75\% hubo lo resultado final de 28 puntos, con alto riesgo de desarrollar úlceras por presión. Después de diez días de hospitalización, 48,3\% de los pacientes no habían recibido ninguna directriz de prevención; 68,3\% de ellos ya tenía úlceras por presión. El uso de la escala de Waterlow permite el control de la asistencia, siendo capaz de influir en los resultados clínicos.

Palabras clave: Enfermería; Úlcera por presión; Tecnología; Escalas. 


\section{INTRODUÇÃO}

Por viver numa era tecnológica, muitas vezes a concepção de tecnologia tem sido usada de forma enfática no cotidiano, porém, equivocadamente, concebida somente como um produto, uma máquina, uma materialidade generalizada e resumida a procedimentos técnicos de operação e seu produto.

A tecnologia, como equipamento, é um componente importante de instrumento de trabalho no exercício educativo, contudo, não se restringe à tecnologia em si ela também está voltada para a organização lógica das atividades, de tal modo que possam ser sistematicamente observadas, compreendidas e transmitidas ${ }^{(1)}$.

Os estudos sobre tecnologia na área da saúde são ainda incipientes, com abordagem da tecnologia médica sob ângulos dos ensaios clínicos, tais como procedimentos diagnósticos, sendo limitada a produção científica que se refere à avaliação tecnológica ${ }^{(2)}$.

A pesquisa em tecnologia de enfermagem é essencial para ensejar a base de conhecimento que fundamenta a prática do enfermeiro, além de poder identificar o impacto do uso da tecnologia nos diversos cenários de atuação de enfermagem. Para entender o contexto atual que reflete a arte do cuidado inserida num mundo tecnológico, é necessário compreender o desenvolvimento histórico e cultural da sociedade ${ }^{(3)}$.

$\mathrm{Na}$ Enfermagem, encontram-se, hoje, diversas formas de tecnologias que podem ser desenvolvidas e especializadas por profissionais motivados em prestar um melhor cuidado à saúde do ser humano.

O gerenciamento do cuidado é praticado nas linhas consideradas estratégicas. Cada uma dessas linhas tem conhecimentos específicos e conta com gestores do cuidado para realizar este trabalho de monitoramento, articulação e integração da assistência ${ }^{(4)}$.

As tecnologias podem ser classificadas em: tecnologia educacional (TE), definida como um conjunto de conhecimentos que tornam possíveis o planejamento, a execução, o controle e o acompanhamento do processo educacional; tecnologia gerencial (TG), considerada como um processo sistematizado e testado de ações teórico-práticas utilizadas no gerenciamento da assistência; e tecnologia assistencial (TA), constituída de um conjunto de ações sistematizadas, processuais e instrumentais para a prestação de uma assistência qualificada ao ser humano em todas as suas dimensões ${ }^{(1)}$.

Acerca das TA, pode-se dizer que estas incluem a formulação de um saber técnico-científico resultante de investigações, aplicações de teorias e da experiência cotidiana dos profissionais e clientela, constituindo-se, portanto, num conjunto de ações sistematizadas, processuais e instrumentais para a prestação de uma assistência qualificada ao ser humano em todas as suas dimensões ${ }^{(5)}$.

Assim sendo, a TA deve possibilitar dimensões interacionais, permitindo aos profissionais a utilização dos sentidos para a escolha e a realização da assistência permitindo encontrar a sensitividade, a solidariedade, o amor, a ética e o respeito de si e do outro (clientela). A TA tem como finalidade apoiar, manter e promover o processo da vida das pessoas em situações de saúde e doença(1).

Desse modo, a enfermagem encontra-se, hoje, com um conjunto de tecnologias que podem cada vez mais ser desenvolvidas e especializadas por todos aqueles profissionais motivados para uma melhoria do cuidado à saúde do ser humano.

A enfermeira deve buscar a formulação do seu próprio conhecimento, um conhecimento que esteja relacionado com a qualidade de vida, a maneira de administrar a saúde, a enfermidade e os problemas daí decorrentes ${ }^{(2,6)}$.

A tecnologia em enfermagem consiste na aplicação sistemática de conhecimentos científicos, com o objetivo de proporcionar melhor atendimento ao ser humano. O enfermeiro, no seu cotidiano, faz uso das tecnologias educacional, gerencial e assistencial, no entanto, apesar de os enfermeiros fazerem uso destas diversas tecnologias em sua prática profissional, eles nem sempre têm consciência deste fato, pois possuem um conhecimento ainda precário sobre esta temática ${ }^{(7-8)}$.

Após alguns estudos envolvendo a temática, surgiu um interesse maior em interligá-la com úlcera por pressão em pacientes com lesão medular. A escolha por pacientes com lesão medular ocorreu por se apresentarem clinicamente susceptíveis ao desenvolvimento da úlcera por pressão, em razão da cronicidade, da gravidade, da média de internação e, sobretudo, pelo elevado grau de dependência.

Diante do exposto este estudo objetivou avaliar a tecnologia de enfermagem utilizada na prevenção de úlcera por pressão na pessoa com lesão medular

\section{METODOLOGIA}

A pesquisa foi desenvolvida em um hospital terciário, especializado no atendimento ao trauma. Para o cálculo amostral foi utilizado o nível de confiança de $95 \%(Z=1,96)$, erro amostral de 5,3\%, numa população de 186 pacientes. Com base nos parâmetros expostos, a amostra foi composta por 60 pacientes, selecionada por conveniência, de forma consecutiva, conforme a internação. Foram considerados como critérios de inclusão: estar internado com diagnóstico médico de lesão medular traumática, ter idade igual ou superior a 18 anos e aceitar participar do estudo, assinando o Termo de Consentimento Informado.

Para a coleta dos dados foi utilizado um roteiro sistematizado de entrevista, construído com a finalidade de obter indicativos de identificação, exame físico, avaliação clínica, orientação sobre úlcera por pressão e aplicação da Escala de Waterlow.

Os resultados foram analisados de forma descritiva e apresentados por meio de tabelas. Os dados foram compilados no software Excel, em forma de planilhas, e a análise estatística pelo programa SPSS versão 13.0. Para a análise de associação dos dados foram utilizados os testes estatísticos de Kolmogorov-Smirnov; o Coeficiente de Correlação de Pearson; e o Coeficiente de Correlação de Spearman.

Foram respeitados os aspectos administrativos e éticos da pesquisa científica, cumprindo as recomendações da resolução 196/96(9), sendo a pesquisa aprovada por Comitê de Ética, com Parecer $n^{\circ}$ 04153/08. 


\section{RESULTADOS}

A Tabela 1 apresenta a caracterização dos pacientes segundo os dados sociodemográficos.

Tabela 1 - Distribuição dos pacientes com lesão medular segundo dados sociodemográficos. Fortaleza-CE, 2008.

\begin{tabular}{|c|c|c|c|c|c|}
\hline \multicolumn{2}{|l|}{ Variáveis } & \multicolumn{2}{|c|}{$\mathrm{N}^{0}$} & \multicolumn{2}{|r|}{$\%$} \\
\hline \multicolumn{6}{|l|}{ Sexo } \\
\hline \multicolumn{2}{|l|}{ Masculino } & \multicolumn{2}{|c|}{49} & \multicolumn{2}{|r|}{81,7} \\
\hline \multicolumn{2}{|l|}{ Feminino } & \multicolumn{2}{|c|}{11} & & 18,3 \\
\hline \multicolumn{6}{|l|}{ Condição conjugal } \\
\hline \multicolumn{2}{|l|}{ Sem companheiro } & \multicolumn{2}{|c|}{35} & & 58,3 \\
\hline \multicolumn{6}{|l|}{ Procedência } \\
\hline \multicolumn{2}{|l|}{ Capital } & \multicolumn{2}{|c|}{33} & \multicolumn{2}{|r|}{55,0} \\
\hline \multicolumn{2}{|l|}{ Interior } & \multicolumn{2}{|c|}{27} & \multicolumn{2}{|r|}{45,0} \\
\hline \multicolumn{6}{|l|}{ Ocupação } \\
\hline \multicolumn{2}{|l|}{ Agricultor } & \multicolumn{2}{|c|}{10} & \multicolumn{2}{|r|}{16,7} \\
\hline \multicolumn{2}{|l|}{ Autônomo } & \multicolumn{2}{|c|}{16} & \multicolumn{2}{|r|}{26,7} \\
\hline \multicolumn{2}{|l|}{ Doméstica } & \multicolumn{2}{|c|}{02} & \multicolumn{2}{|r|}{3,3} \\
\hline \multicolumn{2}{|l|}{ Pedreiro } & \multicolumn{2}{|c|}{09} & \multicolumn{2}{|r|}{15,0} \\
\hline \multicolumn{2}{|l|}{ Funcionário privado } & 09 & 9 & & 15,0 \\
\hline Funcionário público & & 01 & 1 & & 1,6 \\
\hline Aposentado & & 04 & 4 & & 6,7 \\
\hline Desempregado & & 09 & 9 & & 15,0 \\
\hline Religião & & & & & \\
\hline Católica & & 39 & 9 & & 65,0 \\
\hline Evangélica & & 10 & 0 & & 16,7 \\
\hline Outras & & 04 & 4 & & 6,6 \\
\hline Sem religião & & 07 & 7 & & 11,0 \\
\hline Variáveis & $\mathrm{DP}^{1}$ & Mediana & $\mathrm{P}^{25^{2}}$ & $\mathrm{P} 75^{3}$ & K-S(valor p $)^{4}$ \\
\hline 37,12 & 15,146 & 33,00 & 23,00 & 49,00 & 0,187 \\
\hline Escolaridade & 0,98 & 2,0 & 1,0 & 3,0 & 0,001 \\
\hline $\begin{array}{cc}\text { Renda } & \\
\text { Familiar (R\$) } & 439,178\end{array}$ & 387,6467 & 415,00 & 100,950 & 622,500 & 0,051 \\
\hline
\end{tabular}

${ }^{1} \mathrm{DP}$ (desvio padrão). ${ }^{2}$ P25 (percentil 25). ${ }^{3}$ P75 (percentil 75). ${ }^{4} \mathrm{~K}$-S (Teste de Kolmogorov-Smirnov).

Conforme apresentado na Tabela 1, constatou-se o predomínio do sexo masculino (81,7\%). A interferência do indicador "condição conjugal" está relacionada à presença de um(a) companheiro(a) capaz de colaborar para o seguimento da terapêutica recomendada. Consoante se verificou, 41,7\% dos pacientes moravam com companheiro, enquanto 58,3\% não tinham companheiro, um número bastante elevado para quem vai precisar de um cuidador diário.

No que se refere à procedência, 55\% eram da Capital e $42 \%$ do restante do Estado. Em relação à ocupação, (26,7\%) eram autônomos, seguido da profissão agricultor $(16,7 \%)$ e com um número significante de desempregados (15\%). É importante destacar que o autônomo, aqui em Fortaleza-CE, é quase um sinônimo de desempregado, pois não tem salário fixo, trabalha por conta própria e nem sempre tem serviço a ser prestado. Sobre a religião, $81 \%$ pertenciam ao
Tabela 2 - Distribuição das variáveis categóricas e numéricas. Fortaleza-CE, 2008.

\begin{tabular}{|c|c|c|c|}
\hline \multirow{2}{*}{ Variáveis } & \multicolumn{3}{|c|}{ Pontuação } \\
\hline & Média & DP & $\mathbf{p}$ \\
\hline \multicolumn{4}{|l|}{ Sexo } \\
\hline Masculino & 25,00 & 5,552 & $0,322^{3}$ \\
\hline Feminino & 23,18 & 5,6256 & \\
\hline Total & 48,18 & 11,1776 & \\
\hline \multicolumn{4}{|l|}{ Estado Civil } \\
\hline Com companheiro & 25,52 & 5,197 & $0,310^{3}$ \\
\hline Sem companheiro & 24,06 & 5,6255 & \\
\hline Total & 49,58 & 10,8225 & \\
\hline \multicolumn{4}{|l|}{ Acompanhante } \\
\hline Presente & 23,74 & 4,74 & $0,036^{3}$ \\
\hline Ausente & 27,00 & 6,52 & \\
\hline Total & 50,74 & 11,26 & \\
\hline Variáveis & \multicolumn{2}{|c|}{ Coeficiente de Correlação } & \\
\hline Idade & \multicolumn{2}{|c|}{$-0,237^{1}$} & 0,069 \\
\hline Escolaridade & \multicolumn{2}{|c|}{$-0,178^{1}$} & 0,173 \\
\hline Renda familiar & \multicolumn{2}{|c|}{$-0,121^{1}$} & 0,359 \\
\hline $\mathrm{N}^{\circ}$ de filhos & \multicolumn{2}{|c|}{$-0,209^{2}$} & 0,110 \\
\hline Tempo de internação & \multicolumn{2}{|c|}{$0,043^{2}$} & 0,045 \\
\hline Nível da lesão & \multicolumn{2}{|c|}{$0,029^{2}$} & 0,824 \\
\hline
\end{tabular}

${ }^{1}$ Coeficiente Correlação Pearson; ${ }^{2}$ Coeficiente Correlação Spearman; ${ }^{3}$ Teste $T$ supondo variâncias iguais

cristianismo, predominando os católicos, com $65 \%$, seguidos dos protestantes com $16,7 \%$.

Em relação à faixa etária, observou-se uma média de 37,12 anos com um desvio-padrão de 15,146, constatando-se uma população bem jovem numa faixa etária entre 18 e 33 anos, fase de vida ainda bem produtiva, interrompida pela lesão medular. Destes 31 pacientes $(51,6 \%)$ tinham idade inferior a 40 anos.

Quanto à escolaridade, a média de anos de estudo foi 2,13 anos ( $\mathrm{DP}=0,98$, isto é, $\mathrm{DP}=01 \mathrm{ano})$, com 66,7\%, havendo, portanto, um predomínio de pessoas com baixa escolaridade, ou seja, dos que cursaram o ensino fundamental incompleto, $43,3 \%$ sabiam apenas assinar o nome e $26,7 \%$ eram analfabetos. A renda familiar foi avaliada conforme o salário mínimo vigente no Brasil no período da coleta de dados, ou seja, 415,00 reais. A média de salários foi 439.178 reais, com alta dispersão dos valores, predominando pessoas que ganhavam até um salário mínimo $58,3 \%$, uma media salarial baixa. As variáveis escolaridade e renda familiar apresentaram distribuição simétrica (valor $p>0,05$ ).

Com relação ao gênero, os homens apresentaram pontuação discretamente superior às mulheres. Todavia, esta diferença não foi estatisticamente significante. Isto também ocorreu para indivíduos que informaram seu estado civil com companheiro.

Diferença estatisticamente significante $(p=0,036)$ foi identificada entre os pacientes que tinham ou não acompanhante durante o período de internação. A presença de um cuidador contribui para uma média menor de risco para desenvolver úlcera por pressão, embora, pela Escala, tal risco ainda seja considerado altíssimo. 
Entre as variáveis numéricas, apenas a idade e o tempo de internação apresentaram correlação significante com a pontuação. A idade mostrou correlação negativa, indicando que, quanto menor a idade, maior chance de lesão medular e, por conseguinte, maior chance de úlcera por pressão; todavia, esta correlação foi significante somente ao nível de $10 \%$.

O tempo de internação, por sua vez, apresentou correlação positiva significativa ao nível de $5 \%$, revelando que, quanto maior o tempo de internação, maior o risco para desenvolver úlcera por pressão. Aplicando-se o Coeficiente de Correlação de Spearman para o tempo de internação, esta variável mostrou associação estatística, revelando que, quanto maior o tempo de internação, maior o risco para desenvolver úlcera por pressão.

Tabela 3 - Distribuição das variáveis quanto a incidência, ao tempo de desenvolvimento de úlcera por pressão, tempo de internação e orientações recebidas durante a internação hospitalar. Fortaleza-CE, 2008.

\begin{tabular}{llclc}
\hline \multirow{2}{*}{ Úlcera por Pressão } & \multicolumn{2}{c}{ Até 04 dias } & \multicolumn{2}{c}{ Com 10 dias } \\
\cline { 2 - 5 } & $\mathbf{N}$ & $\%$ & $\mathbf{N}$ & $\%$ \\
\hline Apareceu UP & 08 & 13,3 & 41 & 68,3 \\
Não apareceu UP & 52 & 86,7 & 19 & 31,7 \\
Total & 60 & 100 & 60 & 100 \\
\hline \multirow{2}{*}{ Orientações } & \multicolumn{2}{c}{ Antes da UP } & \multicolumn{2}{c}{ Depois da UP } \\
\cline { 2 - 5 } & $\mathbf{N}$ & $\%$ & $\mathbf{N}$ & $\%$ \\
\hline Sim & 06 & 10 & 31 & 51,7 \\
Não & 54 & 90 & 29 & 48,3 \\
Total & 60 & 100 & 60 & 100 \\
\hline
\end{tabular}

Conforme identificado na Tabela 3, em até quatro dias, o número de pessoas que desenvolveram úlcera por pressão foi pequeno em relação ao grande risco que apresentam. Apenas oito destas pessoas (13,3\%) desenvolveram úlcera por pressão. Após uma reavaliação com dez dias, este número cresceu para 41 pessoas $(68,3 \%)$ com desenvolvimento de úlcera por pressão.

Como observado na tabela acima, 48,3\% dos pacientes não receberam qualquer tipo de orientação sobre úlcera
Tabela 4 - Distribuição das variáveis quanto à incidência, ao tempo de desenvolvimento de úlcera por pressão, tempo de internação e orientações recebidas durante a internação hospitalar. Fortaleza-CE, 2008.

\begin{tabular}{lcc}
\hline Tipo de Orientações & N & \% \\
\hline Não recebeu orientação & 29 & 48,3 \\
Mudança de decúbito (MD) & 04 & 6,7 \\
Manter lençóis secos + MD & 12 & 20 \\
Massagem com óleo + MD & 09 & 15 \\
Colchão articulado + MD & 06 & 10 \\
Total & 60 & 100 \\
Tempo de Internação & & \\
Até cinco dias & 01 & 1,7 \\
05 a 10 dias & 06 & 10 \\
10 a 15 dias & 08 & 13,3 \\
15 a 20 dias & 15 & 25 \\
a 30 dias & 20 & 33,3 \\
Mais de 30 dias & 10 & 16,7 \\
Total & 60 & 100 \\
Incidência das UP & & \\
Sacral & 26 & 63,4 \\
Calcâneos & 12 & 29,3 \\
Outros & 03 & 07,3 \\
Total & 41 & 100 \\
\hline
\end{tabular}

por pressão durante a internação hospitalar, 20\% receberam orientação para manter os lençóis secos e mudança de decúbito; $15 \%$ recebeu para fazer massagem com ácidos graxos essenciais e mudança de decúbito; $10 \%$ foram orientados para a compra de colchão articulado ou piramidal; e 6,7\% foram orientados apenas para a mudança de decúbito.

Quanto ao tempo de internação hospitalar, observou-se um tempo de internação hospitalar prolongado onde $25 \%$ dos pacientes teve permanência de 15 a 20 dias; 33,3\% permaneceu de 20 a 30 dias e 10\% uma internação superior a 30 dias. No somatório dos percentuais constata-se que $50 \%$ dos pacientes tiveram uma internação superior a 20 dias, 75\%

Tabela 5 - Distribuição dos fatores biológicos, físicos e mecânicos que contribuem para o aparecimento de úlceras por pressão, segundo a Escala de Waterlow. Fortaleza-CE, 2008.

\begin{tabular}{|c|c|c|c|c|c|c|}
\hline Variáveis & Média & Mediana & $\mathbf{D P}^{*}$ & $\mathbf{P} 25^{* *}$ & $\mathbf{P} 5^{* * *}$ & $\begin{array}{l}\text { K-S }{ }^{* * * *}(\text { valor } \\
\text { p) }\end{array}$ \\
\hline IMC & 0,78 & 0 & 1,260 & 0 & 2,00 & $<0,001$ \\
\hline Tipo de pele & 0,46 & 0 & 0,703 & 0 & 1,00 & $<0,001$ \\
\hline Sexo/Idade & 2,54 & 2,00 & 0,773 & 2,00 & 3,00 & $<0,001$ \\
\hline Continência & 0,75 & 0 & 0,939 & 0 & 2,00 & $<0,001$ \\
\hline Mobilidade & 4,32 & 5,00 & 0,955 & 4,00 & 5,00 & $<0,001$ \\
\hline Subnutrição & 2,58 & 2,00 & 1,796 & 2,00 & 2,00 & $<0,001$ \\
\hline Deficiência neurológica & 5,30 & 6,00 & 1,782 & 6,00 & 6,00 & $<0,001$ \\
\hline Cirurgia de grande porte & 4,90 & 5,00 & 0,548 & 5,00 & 5,00 & $<0,001$ \\
\hline Apetite & 0,98 & 1,00 & 0,841 & 0 & 2,00 & 0,003 \\
\hline Medicação & 5,49 & 4,00 & 2,216 & 4,00 & 8,00 & $<0,001$ \\
\hline Pontuação final & 24,67 & 23,50 & 5,45 & 21,00 & 28,00 & 0,353 \\
\hline
\end{tabular}

${ }^{*}$ DP (Desvio Padrão). ${ }^{* *}$ P25 (percentil 25). ${ }^{* * *}$ P75 (percentil 75). ${ }^{* * *}$ K-S (Teste de Kolmogorov-Smirnov) 
superior a 15 dias e 11,7\% por um período inferior a 10 dias. Relacionado a incidência da úlcera por pressão verificou-se que $63 \%$ das lesões acometiam a região sacral enquanto $30 \%$ estavam distribuídos nos calcâneos e 7\% distribuídos nas outras diversas partes do corpo.

Cada paciente com lesão medular apresentou, em média, $0,78$ de IMC ( $D P=1,260)$, numa pontuação máxima de três pontos. Isto indica que $75 \%$ dos pacientes tinham, no máximo, um escore igual a dois, ou seja, pelo menos $25 \%$ dos pacientes tinham uma pontuação elevada para o IMC. Conforme mostra o Teste de Kolmogorov-Smirnov, o IMC teve distribuição assimétrica $(p<0,001)$.

Quanto à variável tipo de pele, a média foi 0,46 de uma pontuação total de três na Escala de Waterlow; para continência, a média foi 0,75 de uma pontuação total de três; e para apetite, a média foi 0,98 de uma pontuação total de três. Estas variáveis também apresentaram média baixa para fatores biológicos relacionados com o aparecimento de úlcera por pressão e uma distribuição assimétrica.

Ao relacionar a idade e sexo, o item da escala que se refere à soma deste quesito revelou uma pontuação baixa, considerando que $75 \%$ da amostra apresentou um escore até três, de uma soma total de sete pontos.

Avaliando o item mobilidade pela escala de Waterlow, o escore máximo para predizer déficit é de cinco pontos. Os pacientes com lesão medular apresentaram uma média de 4,32 de mobilidade física prejudicada, com desvio-padrão baixo $(0,955)$. Então, $75 \%$ tinham a pontuação máxima de cinco escores, indicando elevado número de pessoas dependentes de cadeira de rodas.

Pertinente à subnutrição celular, que inclui caquexia, insuficiência cardíaca, doença vascular periférica, anemia e tabagismo, a média foi baixa, 2,58 do somatório total de 21 pontos na Escala de Waterlow, visto que uma só pessoa pode apresentar todos os itens no mesmo momento. É um achado importante, por mostrar uma pontuação muito baixa em relação ao total de escores, indicando um baixo risco no tocante a esse item.

$\mathrm{Na}$ deficiência neurológica, avaliam-se especificamente diabetes (quatro pontos) e paraplegia sensitiva ou motora (cinco pontos). O escore máximo para este item é de dez pontos, uma vez que o indivíduo pode ser diabético e apresentar paraplegia ao mesmo tempo. Nos achados, a pontuação média foi $5,30(\mathrm{DP}=1,782)$ e um percentil de 75 com uma pontuação de seis. No entanto, somente quatro pacientes apresentavam diabetes; portanto, considerando apenas o escore cinco, o achado demonstra uma população quase absoluta de paraplegia. Este dado era esperado uma vez que todos os pacientes do estudo são portadores de lesão medular.

Em relação à cirurgia de grande porte, 29 pacientes (48,3\%) não se submeteram a cirurgia. A média desde achado foi 4,90, com percentil de cinco, num escore total de 10 pontos. Percebe-se a predominância dos pacientes que não necessitaram de tratamento cirúrgico. Ao realizar a avaliação da medicação proposta pela Escala de Waterlow, cujo escore máximo é 16 (quatro pontos para cada subitem) percebe-se uma média de 5,49, indicando que $75 \%$ dos pacientes tinham no máximo um escore igual a oito, ou seja, se enquadravam em, pelo menos, dois subitens.
Após aplicar a escala de Waterlow nos pacientes com lesão medular, o escore final apresentou uma média de 24,67 pontos. O percentil de 75 revelou que $75 \%$ dos avaliados apresentou escore final de 28 pontos, ou seja, altíssimo risco para desenvolver úlcera por pressão.

\section{DISCUSSÃO}

Os achados referentes aos fatores demográficos apresentam similaridade com o perfil usualmente descrito para esta população. O baixo nível socioeconômico, a baixa escolaridade e um predomínio de jovens são citados como importantes características de pessoas com lesão medular ${ }^{(10)}$.

É essencial a presença de um companheiro ou pelo menos um cuidador/acompanhante durante a internação hospitalar, considerando a dependência ocasionada pelo déficit neurológico. As mudanças advindas com o trauma raquimedular alteram não apenas a vida dos indivíduos, mas a dinâmica e o relacionamento familiar ${ }^{(11)}$. A associação identificada entre a presença de acompanhante e o risco de desenvolvimento de úlcera por pressão pode ser justificada pelo fato de o acompanhante, muitas vezes, desempenhar um papel de realizar até mesmo atividades de enfermagem, como as mudanças de decúbito, arrumar a cama, auxiliar no banho, alimentar, reposicionar no leito. Apesar disso, com dez dias de internação, mesmo com acompanhante, 41 pacientes desenvolveram úlcera por pressão, indicando uma possível falha de habilidade ou ausência de orientações. Com vistas à promoção da saúde, é indispensável à presença de um cuidador /acompanhante para os pacientes com lesão medular. Entretanto, os cuidadores precisam ser orientados para intervir adequadamente nas situações de cuidado.

No que se refere a lesão medular, é necessário o conhecimento dos aspectos de vida e das dificuldades enfrentadas pelos pacientes e seus componentes familiares para que a enfermeira possa prestar uma assistência com qualidade, atendendo as necessidades de ambos ${ }^{(12)}$.

Em relação aos fatores de risco biológicos, físicos e mecânicos encontrados no paciente com lesão medular após ter aplicado a escala de Waterlow, concluiu-se que a pessoa com lesão medular durante o período de internação tem altíssimo risco para desenvolver úlcera por pressão.

$\mathrm{Na}$ reavaliação do pacientes com dez dias de internação, constatou-se uma predominância de pacientes que desenvolveram úlcera por pressão. No entanto, não houve variação nos dados do índice de massa corporal, permanecendo uma maioria com IMC normal. É provável que, por ser uma população predominantemente jovem, e por tratar-se de acidentes traumáticos e súbitos, estas pessoas encontravam-se saudáveis antes do acidente.

Com a aplicação da Escala ${ }^{(13)}$, os resultados indicaram que os índices estatisticamente significantes para desenvolver UP foram: tipo de pele classificada como não saudável; sexo feminino; idade superior a 50 anos; incontinência fecal; restrição ao leito ou inércia e alteração do apetite.

A análise dos testes de sensibilidade e especificidade para os escores obtidos na última aplicação da escala para os pacientes sem úlcera, e dos obtidos no dia anterior ao 
aparecimento da lesão para os pacientes com úlcera, determinou o escore 15 como o melhor para predizer o desenvolvimento de úlceras de decúbito ${ }^{(14)}$.

A variável mais significativa em relação ao risco para desenvolver úlcera por pressão foi a mobilidade física prejudicada, pois a maioria dos pacientes era dependente de cadeira de rodas ou restrito no leito ${ }^{(14)}$. A imobilidade é, provavelmente, um dos mais importantes fatores de risco para o desenvolvimento da UP porque, similarmente ao paciente que tem diminuição do nível de consciência, o paciente imóvel também não alivia a pressão nas regiões de proeminências ósseas, mantendo, assim, os fatores de intensidade e duração da pressão como a maior causa do desenvolvimento da lesão ${ }^{(15)}$.

A intensidade da pressão em determinada área do corpo quando exagerada, faz com que a pressão dos capilares aumente, causando uma oclusão capilar que acarreta a diminuição do suprimento sanguíneo, de nutrientes e de oxigênio aos tecidos $^{(14)}$. A duração da pressão é um dos principais fatores de significância, pois, quanto maior o tempo de exposição à pressão, maiores serão os danos aos tecidos, uma vez que a tolerância tissular está relacionada à capacidade dos tecidos de suportar essa pressão ${ }^{(16)}$.

Relacionado à subnutrição celular o fator de maior risco neste item avaliado foi a anemia, com 46,3\%. O estado nutricional reduzido prejudica a elasticidade da pele e, a longo prazo, pode levar à anemia e a redução de oxigênio aos tecidos ${ }^{(16)}$. A avaliação nutricional é uma fonte de informação valiosa visto que o estado nutricional é apontado como um dos principais fatores de risco no surgimento das úlceras por pressão.

Em relação a orientações, quase a totalidade dos pacientes não receberam qualquer tipo de informação antes do advento da úlcera por pressão e mesmo após o aparecimento da úlcera por pressão somente metade recebeu algum tipo de orientação. A orientação de mudança de decúbito estava na maioria das vezes associadas a outras orientações como manter os lençóis secos, massagens com óleos e compra de colchão articulado.

Nos últimos anos atribuiu-se ênfase ao estudo da úlcera por pressão, com a utilização de escalas para predizer os riscos e o tratamento das lesões ${ }^{(17)}$.

É antiga a discussão, a preocupação e o desafio para evitar as úlceras por pressão, mas pouco se tem evoluído no trabalho com a implantação e operacionalização para prevenção das úlceras por pressão. Desta forma, a enfermeira deve proporcionar aos pacientes um cuidado sem dicotomia assistencial/científica e com o máximo de qualidade possível, porquanto, além das incapacidades geradas pela lesão medular, estes, na grande maioria, são jovens, já em processo de perdas de planos e projetos, em virtude das sequelas deixadas pelo trauma.

A importância da orientação para prevenir ulcera por pressão é um importante papel exercido pelo enfermeiro que deve envolver também a equipe de enfermagem e familiares. Nessa ótica observa-se a importância da enfermeira desempenhar atividades de educação em saúde ao familiar cuidador com o intuito de prevenir as úlceras por pressão ${ }^{(18)}$.

O estudo demonstra que a aplicação da escala de Waterlow é eficaz e sensível para avaliar os fatores de risco para úlceras por pressão em pessoas hospitalizadas por lesão medular, direcionando o cuidado supracitado.

\section{CONCLUSÕES}

Evidenciou-se no estudo que a aplicação da escala de Waterlow torna-se eficaz e sensível para avaliar os fatores de risco que contribuem para o aparecimento de úlceras por pressão em pessoas hospitalizadas acometidas por lesão medular.

Embora se conceitue tecnologia em enfermagem como aplicação sistemática de conhecimentos científicos à facilitação do processo de melhor atender o ser humano evidencia-se o fato de que as técnicas com evidências científicas na enfermagem valorizam a profissão.

Ressalta-se que a presença de indicadores nutricionais é uma condição ímpar para se atestar a qualidade e abrangência desta ferramenta de predição na aplicação da escala. Porém quando a instituição não dispõe de camas metabólicas, que permitem pesar e medir o paciente acamado, este item torna-se um fator de dificuldade para operacionalização da escala.

Constatou-se que o fator de risco mais significante para o desenvolvimento da úlcera por pressão na pessoa acometida por lesão medular relaciona-se com a mobilidade física prejudicada, associada ao déficit neurológico instalado.

A presença de um cuidador tem fundamental importância sendo necessário que a equipe de saúde, especialmente a enfermeira, forneça orientações objetivando sua colaboração na prevenção das úlceras no âmbito hospitalar e domiciliar posteriormente visando a promoção da saúde do paciente como também do familiar cuidador.

\section{REFERÊNCIAS}

1. Nietsche EA, Backes VMS, Colomé CLM, Ceratti RN. Tecnologias educacionais, assistenciais e gerenciais: uma reflexão a partir da concepção dos docentes de enfermagem. Rev Latino-am Enfermagem 2005;13(3):344-53.

2. Nietsche EA. Tecnologia emancipatória: possibilidade para a práxis de enfermagem. Unijuí (RS): Unijuí; 2000.

3. Rocha PK, Prado ML, Wal ML, Carraro TE. Cuidado e tecnologia: aproximações através do Modelo de Cuidado. Rev Bras Enferm 2008;61(1):113-6.
4. Malta DC, Jorge ADO. Modelos assistenciais na saúde suplementar: o caso de uma operadora de autogestão no Brasil. Ciênc Saúde Coletiva 2008;13(5):1535-42.

5. Mendes I, Carvalho MD, Almeida RT, Moreira ME. Uso da tecnologia como ferramenta de avaliação no cuidado clínico de recém-nascidos prematuros. J Pediatr 2006; 82(5):371-6.

6. Macedo PO, Quitete JB, Lima EC, Santos ID. As tecnologias de cuidado de enfermagem obstétrica fundamentadas 
pela teoria ambientalista de Florence Nightingale. Esc Anna Nery R Enferm 2008;12(2):341-7.

7. Campos ADCS, Cardoso MVLML. Tecnologia educativa para a prática do cuidado de enfermagem com mães de neonatos sob fototerapia. Texto Contexto Enferm 2008; 17(1):36-44.

8. Martins CR, Dal Sasso GTM. Tecnologia: definições e reflexões para a prática em saúde e enfermagem. [editorial]. Texto Contexto Enferm 2008;17(1):11-2.

9. Ministério da Saúde (BR). Conselho Nacional de Saúde, Comissão Nacional de Ética em Pesquisa. Resolução $N^{\circ}$ 196 de 10 de outubro de 1996: aprova as diretrizes e normas regulamentadoras de pesquisa envolvendo seres humanos. Brasília: Ministério da Saúde; 1996.

10. Dawodu ST. Spinal cord injury: definition, epidemiology, pathophysiology. Med J [serial online] 2001 [cited 2007 Ago 16];2(8):[04 screens]. Available from:http:// www. emedicine.com/pmr/topic182.htm

11. Venturini DA, Decesaro MN, Marcon SS. Conhecendo a história e as condições de vida de indivíduos com lesão medular. Rev Gaúcha Enferm 2006;27(2):219-29.

12. Barbosa IV. Lesão medular e suas significações para a família [dissertação]. Fortaleza (CE): Departamento de Enfermagem, Universidade Federal do Ceará; 2008.

13. Rocha $\mathrm{ABL}$, Barros $\mathrm{SMO}$. Avaliação de risco de úlcera por pressão: propriedades de medida da versão em português da escala de Waterlow. Acta Paul Enferm 2007;20(2):143-50.

14. Rocha ABL. Tradução para a língua portuguesa, adaptação transcultural e aplicação clínica da escala de Waterlow para avaliação de risco de desenvolvimento de úlcera de decúbito [dissertação]. São Paulo (SP): Escola Paulista de Medicina, Universidade Federal de São Paulo; 2003.

15. Paranhos WY. Úlceras de pressão. In: Silva AJ, Dantas SRPE. Abordagem multiprofissional do tratamento de feridas: São Paulo: Atheneu; 2003. p. 287-97.

16. Dealey C. Cuidando de feridas. São Paulo: Atheneu; 2008.

17. Fernandes LM. Úlcera de pressão em pacientes críticos hospitalizados uma revisão integrativa da literatura. [dissertação]. Ribeirão Preto (SP): Escola de Enfermagem de Ribeirão Preto, Universidade de São Paulo; 2004.

18. Leite VBE, Faro ACM. Identificação de fatores associados às úlceras por pressão em indivíduos paraplégicos relacionados ás atividades de lazer. Acta Fisiatr 2006;13(1):21-5. 